\title{
The fruitful coexistence of Borrelia burgdorferi and Ixodes ticks
}

\author{
Dominik LeWANdowski ${ }^{1}$, ANNa UrbanowiCZ ${ }^{1}$, Marek Figlerowicz ${ }^{1,2 *}$ \\ ${ }^{1}$ Institute of Bioorganic Chemistry, Polish Academy of Sciences, Poznań, Poland \\ ${ }^{2}$ Institute of Computing Science, Poznan University of Technology, Poznań, Poland \\ *Corresponding author: marekf@ibch.poznan.pl
}

Over the last decade, the incidence of tick-borne diseases in Europe has increased rapidly. In particular, Lyme disease cases have been found to occur frequently. The disease, also called borreliosis, is caused by Borrelia burgdorferi sensu lato (s. l.) spirochetes. The life cycle of this pathogen involves both a vertebrate host and an Ixodes tick vector. The Ixodes ricinus species is a dominant vector for $B$. burgdorferi s. 1 . in Poland and in other European countries. Recent studies indicate that from a few percentage points to approximately $40 \%$ of the European tick population are infected with $B$. burgdorferi, depending on the region (Chmielewska-Badora et al., 2012; Welc-Faleciak et al., 2010). Borreliosis in humans is a serious chronic disease affecting multiple organ systems, including the nervous system, cardiovascular system, muscles and joints. However, humans are only accidental hosts for Borrelia spirochetes. In the natural environment the reservoir of Borrelia are small vertebrates, mainly rodents (Franke et al., 2013).

B. burgdorferi enters a tick when the latter is feeding on an infected vertebrate. Ticks have developed complex mechanisms enabling them to overcome the host's defense systems. Interestingly, Borrelia spirochetes have gained the capacity to use these mechanisms for their own purposes. To survive in the vector and to enter the host, Borrelia utilizes both its own lipoproteins anchored in the external bacterial membrane (outer surface proteins) and the tick-produced proteins. Once the Borrelia penetrates into a tick's gut, the expression of bacterial outer surface proteins (Osp) A and $\mathrm{B}$ is up-regulated. It is postulated that both proteins (OspA and OspB) permit pathogens to stick to the vector's epithelial cells (de Silva et al., 1996). At least one tick protein (TROSPA from I. scapularis) has been proven to be a molecular determinant of the colonization of the vector body by direct interaction with OspA (Pal et al., 2004a).

After consuming blood, the immature form of the tick detaches from the host and moults into a subsequent developmental form. During the long periods between tick moulting, the Borrelia is exposed to starvation and oxidative stress. Bacteria try to avoid these disadvantageous conditions by producing a new set of proteins. It has been shown that among the genes activated in spirochetes during starvation is one encoding the BB0690 protein. BB0690 is homologous to a starvationinducible DNA-binding protein (Dps) from $E$. coli. Dps protects bacterial DNA against damage during periods of famine. The borrelial homolog of Dps forms a dodecameric complex capable of sequestering iron to prevent reactions which generate reactive oxygen species. BB0690 has been proven to be essential for bacterial persistence in dormant ticks (Li et al., 2007). Other bacterial proteins, such as BptA (borrelial persistence in tick protein A) or lipoprotein La7 (annotated as BB0365) have also been shown to be required for Borrelia survival in ticks. However, the molecular mechanisms underlying the activity of these proteins remain to be elucidated (Revel et al., 2005; Pal et al., 2008). Another lipoprotein, $\mathrm{BB} 0323$, found in the borrelial outer membrane has been demonstrated to be necessary for both the survival of the bacteria in the vector and the transfer of the pathogen from the tick to the host organism. Although it has been shown that spirochetes lacking BB0323 are clumped together and have disintegrated outer membranes, the specific role of BB0323 in spirochete biology remains unknown (Zhang et al., 2009). After glucose depletion in the tick's gut, Borrelia needs 
to adapt to nutritional changes, including carbon source. To this end, the $g l p$ operon is activated. It contains genes coding for glycerol metabolic enzymes. Thus, bacteria can capture and utilize glycerol molecules produced by overwintering ticks (Pappas et al., 2011).

During subsequent tick engorgement, the conditions in the gut significantly change. For the spirochetes this change is a signal for an opportunity to colonize a new host. As a result, $B$. burgdorferi alters its gene expression pattern, decreasing $o s p A / B$ expression, whereas the expression of $\operatorname{csp} C$ is increased. This change facilitates dissemination of Borrelia from the tick's gut towards the salivary glands (Pal et al., 2004b). Migration of spirochetes through the tick's body is not a wellknown process. It is postulated that interactions between bacterial surface protein BBE31 and the TRE31 protein secreted by tick epithelial cells (Zhang et al., 2011), as well as between host-derived plasminogen and OspA, could play a role in the process (Coleman et al., 1997). In salivary glands, bacteria bind with tick protein - Salp15 (Salivary protein 15kDa). Bacterial surface protein OspC is involved in this interaction (Tilly et al., 2006). Salp15-OspC binding enables spirochetes to evade the host immunological response (Ramamoorthi et al., 2005; Schuijtet al., 2008). Salp15 has been proven to possess immunosuppressive properties by inhibiting $\mathrm{CD}^{+}$T-cell activation (Anguita et al., 2002). Furthermore, interaction between OspC and Salp15 could be beneficial to both spirochete and tick because Borrelia upregulates Salp15 expression in the blood-sucking tick, which can contribute to more effective feeding (Ramamoorthi et al., 2005). Salp20 from I. scapularis has also been proposed as a facilitator of tick feeding and possibly a protector of $B$. burgdorferi from complement-mediated killing (Tyson et al., 2007). Another tick salivary protein that plays a crucial role in the acquisition of $B o r$ relia by the vector is Salp25D. It functions as an antioxidant, detoxifying reactive oxygen species at the vector-pathogen-host interface (Narasimhan et al., 2007).

In response to skin damage and tick antigens, the host produces a wide spectrum of factors involved in a non-specific immune response. The tick tries to overcome this first line of defense by producing a great number of anticoagulants, blood vessel relaxants, anti-inflammatory and immunomodulatory factors. All of them are present in tick saliva (Valenzuela et al., 2002). Certainly, all of these compounds significantly facilitate the inva- sion of a host by tick-borne bacteria. It has been found that the soft tick produces lipocalin which blocks both classical and alternative complement activation pathways (Nunn et al., 2005). Extract from the tick salivary glands inhibits the secretion of inflammatory cytokines by blood cells (Ramachandra et al., 1992). Moreover, it has been demonstrated that hard tick saliva could inhibit the activity of neutrophils and deactivate anaphylatoxins (Francischetti et al., 2009), as well as block the angiogenesis process (Fukumoto et al., 2006).

Inhibition of the host reaction causing pain and itching may be one of the most important factors determining the success of a tick parasitic strategy. One of the pain mediators is ATP released from the damaged cells, as well as serotonin and histamine released by platelets and mast cells. It has been shown that tick saliva contains enzymes that degrade ATP (Francischetti et al., 2009) and lipocalin SHBP (Serotonin and Histamine Binding Protein) which traps both serotonin and histamine (Sangamnatdej et al., 2002).

Tick-Borrelia interaction is a very interesting and complex example of parasitism. B. burgdorferi is known to be adapted to the vector in such a manner that it is capable of employing tick strategies to increase its infection capacity (Radolf et al., 2012; Franke et al., 2013; Lewandowski et al., 2013). Better understanding of the mechanisms underlying the phenomenon is indispensable for elaborating effective strategies for Lyme disease prophylactics and treatment.

\section{References}

Anguita J., Ramamoorthi N., Hovius J.W., Das, S., Thomas V., Persinski R., Conze D., Askenase P.W., Rincon M., Kantor F.S., Fikrig E. (2002) Immunity 16: 849-859.

Chmielewska-Badora J., Moniuszko A., Zukiewicz-Sobczak W., Zwolinski J., Piątek J., Pancewicz S. (2012) Ann. Agric. Environ. Med. 19: 271.

Coleman J.L., Gebbia J.A., Piesman J., Degen J.L., Bugge T.H., Benach J.L. (1997) Cell 89: 1111-1119.

Dai J., Wang P., Adusumilli S., Booth C.J., Narasimhan S., Anguita J., Fikrig E. (2009) Cell Host Microbe 6: 482-492. de Silva A.M., Telford S., Brunet L.R., Barthold S.W., Fikrig E. (1996) J. Exp. Med. 183: 271-275.

Francischetti I.M., Sa-Nunes A., Mans B.J., Santos I.M., Ribeiro J.M. (2009) Front. Biosci. 14: 2051-2088.

Franke J., Hildebrandt A., Dorn W. (2013) Ticks Tick Borne Dis. 4: $11-25$.

Fukumoto S., Sakaguchi T., You M., Xuan X., Fujisaki K. (2006) Microvasc. Res. 71: 218-221. 
Lewandowski D., Urbanowicz A., Figlerowicz M., (2013) Post. Mikrobiol. 52: 9-16.

Li X., Pal U., Ramamoorthi N., Liu X., Desrosiers D.C., Eggers C.H., Anderson J.F., Radolf J.D., Fikrig E. (2007) Mol. Microbiol. 63: 694-710.

Narasimhan S., Sukumaran B., Bozdogan U., Thomas V., Liang X., DePonte K., Marcantonio N., Koski R.A., Anderson J.F., Kantor F., Fikrig E. (2007) Cell Host Microbe. 2: 7-18.

Nunn M.A., Sharma A., Paesen G.C., Adamson S., Lissina O., Willis A.C., Nuttall P.A. (2005) J. Immunol. 174: 2084-2091.

Pal U., Dai J., Li X., Neelakanta G., Luo P., Kumar M., Wang P., Yang X., Anderson J.F., Fikrig E. (2008) J. Infect. Dis. 197: 148-155.

Pal U., Li X., Wang T., Montgomery R.R., Ramamoorthi N., de Silva A.M., Bao F., Yang X., Pypaert M., Pradhan D., Kantor F.S., Telford S., Anderson J.F., Fikrig E. (2004a) Cell 119: 457-468.

Pal U., Yang X., Chen M., Bockenstedt L.K., Anderson J.F., Flavell R.A., Norgard M.V., Fikrig E. (2004b) J. Clin. Invest. 113: 220-230.

Pappas C.J., Iyer R., Petzke M.M., Caimano M.J., Radolf J.D., Schwartz I. (2011) PLoSPathog. 7(7): e1002102.
Radolf J.D., Caimano M.J., Stevenson B., Hu L.T. (2012) Nat. Rev. Microbiol. 10: 87-99.

Ramachandra R.N., Wikel S.K. (1992) J. Med. Entomol. 29: 818-826.

Revel A.T., Blevins J.S., Almazan C., Neil L., Kocan K.M.., de la Fuente J., Hagman K.E., Norgard M.V. (2005) Proc. Natl. Acad. Sci. USA. 102: 6972-6977.

Sangamnatdej S., Paesen G.C., Slovak M., Nuttall P.A. (2002) Insect Mol. Biol. 11: 79-86.

Tilly K., Krum J.G., Bestor A., Jewett M.W., Grimm D., Bueschel D., Rosa P. (2006) Infect. Immun. 74: 3554-3564.

Tyson K., Elkins C., Patterson H., Fikrig E., de Silva A. (2007) Insect Mol. Biol. 16: 469-479.

Valenzuela J.G., Francischetti I.M., Pham V.M., Garfield M.K., Mather T.N., Ribeiro J.M. (2002) J. Exp. Biol. 205: 28432864.

Welc-Faleciak R., Hildebrandt A., Sinski E. (2010) Ann. Agric. Environ. Med. 17: 309-313.

Zhang L., Zhang Y., Adusumilli S., Liu L., Narasimhan S., Dai J., Zhao Y.O., Fikrig E. (2011) PLoS Pathog. 7(6): e1002079.

Zhang X., Yang X., Kumar M., Pal U. (2009) J. Infect. Dis. 200: 1318-1330. 